\title{
Sample refreshment schemes for high repetition rate FEL experiments
}

Joachim Schulz Sadia Bari Jens Buck Charlotte Uetrecht 


\title{
Sample refreshment schemes for high repetition rate FEL experiments
}

\author{
Joachim Schulz, Sadia Bari, Jens Buck and Charlotte Uetrecht \\ European XFEL GmbH, Notkestr. 85, 22607 Hamburg, Germany
}

\begin{abstract}
Free-electron lasers offer a variety of unique properties for spectroscopy and imaging. The combination of high peak brilliance and a high repetition rate opens a window to experiments that have not been feasible so far but also introduces challenges in sample preparation and refreshment. First experiments at the Linac Coherent Light Source (LCLS) in Stanford showed the potential of free electron lasers for serial X-ray crystallography as well as for imaging non-reproducible objects. Owing to the superconducting accelerator technology, the European X-ray Free-Electron Laser Facility (European XFEL) will allow an average repetition rate of up to $27 \mathrm{kHz}$ with bunch separation in the order of $200 \mathrm{~ns}$. This extremely high repetition rate gives great chances for the scientific impact of the European XFEL, but it also comes with challenges for providing fresh samples for each bunch. This contribution will give an overview of the sample environment techniques that are in consideration for the European XFEL Facility. These techniques include gas phase, liquid, and aerosol sources for life science and physics experiments.
\end{abstract}

Keywords: XFEL sample delivery, liquid jets, aerodynamic lens, mass spectrometry, aerosols

\section{INTRODUCTION}

X-ray free-electron laser (XFEL) sources are valuable devices because of their high peak brightness and ultra short pulse lengths that allow resolving chemical processes in the femtosecond range. Because of the statistical nature of the X-ray production in SASE* XFELs, a typical experiment consists of many repetitions of identical measurements. A common data analysis method is to sort the data according to X-ray energy and pulse intensity and then average the data taken with similar beam parameters. This technique has the strength of processing a whole range of energies and intensities simultaneously. Thereby it inherently avoids systematic errors due to drift that occur when data is taken with a scanning source.

To study time dependent processes, pump-probe-schemes with an external laser as excitation source and the XFEL pulses as probe are common approaches. In this technique, the unavoidable jitter in the synchronization between laser light and X-rays induces another degree of freedom. The time resolution can be considerably increased by sorting the data with respect to an external relative timing measurement. ${ }^{1}$

The need to select data taken with similar beam parameters out of a plethora of different measurements sets the demand for high repetition rates to collect enough data in a reasonable time. High repetition rates, on the other hand, set high demands on the equipment for XFEL experiments. The detectors need to read out fast enough to meet the repetition rate of the X-ray source. This causes high data rates that have to be pre-processed in real time and stored for future evaluation. Online data has to be extracted for the scientists to control and optimize the experiment. Finally, the high intensity of the X-rays in XFEL sources demands to change the sample rapidly. In many experiments, the sample is destroyed in a single pulse of the XFEL and has to be replaced before the next pulse.

Table 1 gives an overview over the XFEL sources and their repetition rates. Two time scales are important for sample refreshment: The normal conducting sources reach repetition rates of $120 \mathrm{~Hz}$, allowing about $8 \mathrm{~ms}$ time between subsequent pulses for sample refreshment. The superconducting accelerator of the European XFEL

Further author information: (Send correspondence to J.S.)

J.S.: E-mail: Joachim.Schulz@xfel.eu, Telephone: +49 4089984511

*self amplified spontaneous emission

Advances in X-ray Free-Electron Lasers II: Instrumentation, edited by

Thomas Tschentscher, Kai Tiedtke, Proc. of SPIE Vol. 8778, 87780T · (c) 2013

SPIE · CCC code: 0277-786X/13/\$18 · doi: 10.1117/12.2019754

Proc. of SPIE Vol. 8778 87780T-1 
Table 1. Repetition rate of XFEL sources

\begin{tabular}{|l|c|l|}
\hline Source & Start & Repetition rate \\
\hline LCLS (Stanford, USA) & 2009 & $30 \mathrm{~Hz}, 60 \mathrm{~Hz}, 120 \mathrm{~Hz}$ \\
\hline SACLA (Hyogo, Japan) & 2011 & $\max 60 \mathrm{~Hz}$ \\
\hline European XFEL (Schenefeld, Germany) & 2016 & $\begin{array}{l}4.5 \mathrm{MHz} \text { bunch trains in } 10 \mathrm{~Hz} \\
27000 \text { bunches per second }\end{array}$ \\
\hline
\end{tabular}

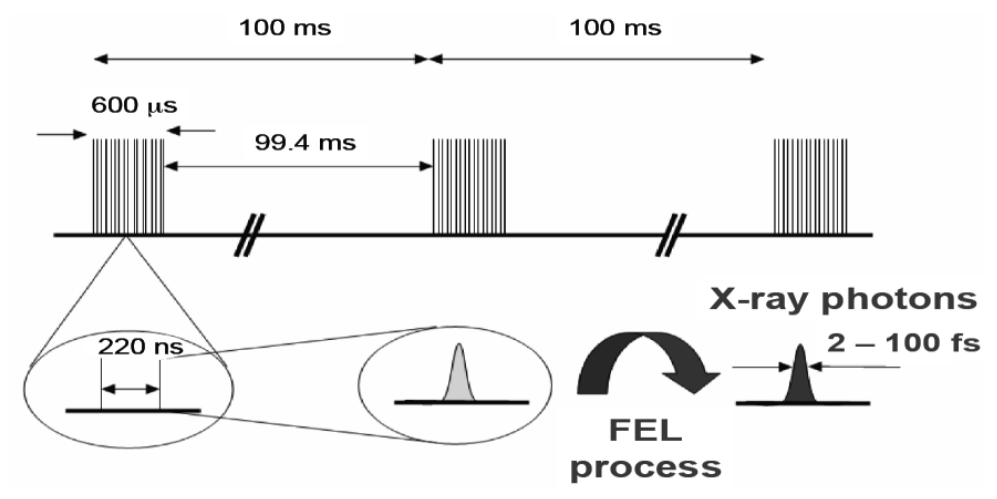

Figure 1. The pulse structure of the European XFEL Facility. Electron bunches will be acellerated in bunch trains of up to 2.700 bunches with a bunch separation corresponding to $4.5 \mathrm{MHz}$. The bunch trains will be produced with a $10 \mathrm{~Hz}$ repetition rate. (Figure from European $\mathrm{XFEL}^{2}$ )

Facility will allow for production of up to $27.000 \mathrm{X}$-ray pulses per second. These pulses will come in bunch trains with a repetition rate of $10 \mathrm{~Hz}$. Within each pulse train, 2.700 pulses can be generated with a repetition rate of $4.5 \mathrm{MHz}$. A complete filling of this pulse structure leaves only $200 \mathrm{~ns}$ time for sample refreshment between subsequent pulses. However, it leaves $99 \mathrm{~ms}$ between subsequent bunch trains. A scheme of the pulse structure is given in figure 1.

The unique pulse structure of the European XFEL delivers over 200 times as many pulses per second as an XFEL based on normal conducting accelerators. The 2D detector technology (for example the Adaptive Gain Integrating Pixel Detector $\mathrm{AGIPD}^{3}$ or the Large Pixel Detector $\mathrm{LPD}^{4,5}$ ) will be able to collect 2.000 to 5.000 images per second. Thus, a typical experiment at the European XFEL can be about 20 times faster. Within 20 min, it has the potential to collect as much data as a $120 \mathrm{~Hz}$ source in an $8 \mathrm{~h}$ shift. An experiment that takes minutes rather than hours sets high demands on reliability and robustness of sample delivery methods.

\section{SAMPLE TYPES}

Experiments at XFELs use a large variety of different samples. For the first gas phase measurements at a soft X-ray FEL, the TESLA Test Facility (TTF) at DESY in Hamburg (Germany), a rare gas cluster source has been used. ${ }^{6}$ This source was capable of producing pulsed supersonic jets of rare gas atoms as well as clusters. Similar experiments on free atoms ${ }^{7}$ and rare gas clusters ${ }^{8}$ provide exciting new results at the hard X-ray FELs.

In this report, we focus on sample delivery methods that are fit to bring biological samples into the beam of an FEL. The potential of XFEL sources for biomolecular imaging has been pointed out in 2000 by Richard Neutze et $a l .{ }^{9}$ In the imaging before destruction approach, a biomolecule can scatter enough photons for a complete reconstruction of the shape, before it is destroyed by the absorbed energy. The principle has been demonstrated on a fixed target diffraction pattern at the soft X-ray FEL FLASH in Hamburg. ${ }^{10}$

At the XFEL LCLS in Stanford (USA), the goal to image biomolecules has been approached from two sides. Serial femtosecond nano-crystallography ${ }^{11}$ can solve the structure of protein complexes in nanosized crystals. Growing large crystals for crystallography at synchrotron radiation sources is the main bottleneck in structural biology. Serial nano-crystallography can overcome this problem by recording the scattering of hundred thousands 
of randomly oriented nano-crystals and computationally reconstructing the 3D-structure. By this method, a new structure has been solved recently. ${ }^{12}$ The other looks at single particles of large biological objects such as viruses. ${ }^{13}$ For X-ray serial nano-crystallography, the crystals are prepared in liquid suspension and brought into the X-rays by liquid jets. The enhancement of the scattering amplitude in the Bragg-peaks is strong enough to exceed the background from scattering in the solvent. Imaging of single virus particles on the other hand relies on free particles in vacuum. Here aerosol sources are necessary.

\section{LIQUIDS}

\subsection{Liquid jets}

Liquid jet systems can produce stable streams of liquids and particles in solution. Mainly two types of liquid jet nozzles are used in cross beam experiments with synchrotron or XFEL light: Pure liquid nozzles (Rayleigh jets) and liquid nozzles assisted by coaxial gas flow also referred to as gas dynamic virtual nozzle (GDVN).

Vacuum compatible systems of the Rayleigh jets with diameters ranging approximately from 5 to $100 \mu m$ exist. These nozzles are applied e.g. for photoelectron and X-ray absorption spectroscopy. ${ }^{14,15}$ These nozzles are also used with the soft laser desorption technique where ions are laser desorbed from liquid beams or droplets to study biological macromolecules by mass spectrometry. ${ }^{16}$ Here, typical liquid jet flow velocities are up to 120 $\mathrm{m} / \mathrm{s}$ driven by a chromatography system with pressures at 10-50 bar. The sample consumption is in the $\mathrm{ml} / \mathrm{min}$ range. ${ }^{17}$

For delivering biologically relevant material, such as nano-crystals to the interaction region, the sample consumption of these conventional jets is too high. Sample consumption can be reduced by slowing down the stream or - more effectively - by reducing the diameter of the nozzle. However, for orifice diameters smaller than $10 \mu \mathrm{m}$, clogging of the nozzles becomes an issue. To reduce the jet diameter without the danger of clogging the sample delivery, GDVN liquid jets were introduced. ${ }^{18-20}$ The liquid jet flows through a central capillary and is focused down to a few micrometers by the pressure of co-flowing gas when passing through an aperture. Typically the central capillary has an inner diameter of $50 \mu \mathrm{m}$, centered inside a glass tube, which is flame polished at the end to form a convergent 50-100 $\mu \mathrm{m}$ diameter. Liquid and gas pressures are in the order of 15 and 30 bar respectively. ${ }^{20}$ Even jet diameters below $1 \mu \mathrm{m}$ have been recently achieved. ${ }^{21}$ The state of the art gas dynamic virtual nozzles are difficult to manufacture. New designs, like using microfluidic devices for mass production of nozzles, are under consideration.

GDVN liquid jets have been successfully used for nano crystallography ${ }^{11}$ at different XFELs. These liquid jets have the advantage to study biological samples in their natural environment and to refresh the sample throughout the experiment with sample consumption in the order of $10 \mu \mathrm{l} / \mathrm{min}^{20}$

In general, the liquid jet emerges from the nozzles as a cylindrical stream, which eventually breaks up into droplets due to the Rayleigh instability. The droplets can be used as an aerosol source for an aerodynamic lens.

Online monitoring of the liquid jets is fundamental to control their performance, i.e. stable jetting, no clogging of the nozzle or freezing of the system. Furthermore, online monitoring is an important tool for checking the overlap of the liquid jet with the X-ray beam or with a pump laser by visualization of the plasma or illumination respectively.

A lot of technical developments have improved the application of liquid jets under vacuum conditions. To avoid a too high gas load from the evaporating solvent, the free jet in vacuum has to be as short as possible. To avoid contamination of the detector, a catcher system needs to accept the biggest part of the solvent and ideally all the suspended specimen.

Today, two different designs for a catcher system exist. One design consists of a nozzle shroud and a catcher shroud connected with a bayonet coupling to make connection without breaking the vacuum possible. The nozzle shroud is differentially pumped with a small inlet hole for the FEL beam and a wide exit angle for scattered light. Removing the nozzle without breaking the vacuum of the main chamber is also possible. The catcher shroud consists mainly of a turbo pumped tube. Under liquid jet operation the main chamber pressure stays below $4 \cdot 10^{-5}$ mbar. $^{20}$ 
The second design is used with the Rayleigh jets. A beam trap, a little container, collects the liquid. The dimensions of the skimmer entrance channel of the beam trap are chosen under consideration of the hydrodynamic forces between the incoming liquid and the outflowing vapor gas. Heating of the catcher aperture helps preventing ice formation and drives the condensing liquid to a cooled recycling vessel. Also here pressures of $10^{-5}$ mbar can be achieved in the main chamber. ${ }^{22}$

Additionally sample delivery to the liquid jets needs also carefully consideration. For example the system should be light tight and temperature controlled to make sure that temperature or light sensitive samples are not destroyed. A rotating sample delivery device has been built, currently also temperature controlled, to prevent settling of crystals in the liquid. ${ }^{23}$

\subsection{Liquid sheets}

For some applications it is preferable to use a flat liquid sheet rather then a cylindrical jet. Those liquid sheets have a better defined surface so that the incident angle of the radiation can be easily controlled. Also the thickness of the sheet is better defined compared with a round jet. This can be helpful for absorption measurements.

Liquid sheets can be formed in different ways: With a flat spray nozzle ${ }^{24}$ with a liquid jet impact on a disk ${ }^{25}$ and with two impinging jets. ${ }^{26}$ Already in 1833, Savart started his interest in liquid sheets. Surface tension and dynamic and static properties were investigated. Later, more details of controlling the liquid atomization in engines were of interest. ${ }^{27}$

\subsection{Towards higher efficiency}

The special time structure of the European XFEL Facility (see figure 1) makes pulsing of liquid jet sources desirable. While for the normal conducting XFELs, slowing down the jet is an effective way of increasing sample delivery efficiency, for the European XFEL Facility the jet needs to deliver a fresh sample in only 220 ns within a pulse train of $600 \mu s$ length. For the rest of $100 \mathrm{~ms}$, no X-ray pulses are available. Therefore a method is necessary to deliver samples effectively in a pulsed mode.

A straight forward method for pulsed sample delivery method would be a drop-on-demand source as they are used for ink-jet printer heads. However, biosamples in suspension tend to settle and clog the nozzle when the stream stops. Another approach will be on-chip mixing of liquids in microfluidic devices. Here the samples could be mixed into a constantly floating stream of solvent synchronized with the XFEL source. Future investigations have to show the feasibility and the prospects of the different approaches.

\section{AEROSOL INJECTORS}

Aerosol injectors allow to introduce any kind of aerosol particles or droplets into the vacuum of the sample chamber. Examples for aerosol sources include the afore-mentioned liquid jets and spark generators that produce an aerosol of soot particles. ${ }^{28,29}$ For a user facility like the European XFEL, this versatility is a major advantage. The same aerosol injector, combined with a variety of aerosol sources, can be used by many user groups to inject their sample into the interaction region.

Compared with particles in liquid jets or on a substrate, aerosol particles are free from surrounding scatterers in the solvent or substrate. Therefore the image is considerably cleaner and the reconstruction algorithm ${ }^{28,30} \mathrm{can}$ be based on the assumption of a finite scatterer. This kind of prior knowledge can be important to reconstruct the real space image from the scattered intensities. In this section, we will first introduce the concept of aerosol injectors in general and then suggest the use of native mass spectrometry in order to make more efficient use of the sample. 


\subsection{Aerodynamic lenses}

In an aerodynamic lens injector, an aerosol streams through a cascade of apertures. These apertures act as aerodynamic lenses, concentrating the particle density in the center of the stream. The concept has been described by Murphy and Sears in 1964. ${ }^{31}$ Liu and co-workers developed a more complete description of the theoretical foundations ${ }^{32}$ and tested the dependence of the beam profile in dependence on the most important parameters experimentally. ${ }^{33}$

The focussing of the aerosol particles and droplets is governed by the particle Stokes number

$$
S=\frac{\tau_{0} U_{0}}{d_{f}}
$$

where $\tau_{0}$ is the particle relaxation time, $U_{0}$ and $d_{f}$ are the fluid velocity and the inner lens diameter, respectively. The particle relaxation time is a measure, how quickly the aerosol particles follow direction and velocity changes of the fluid. It is generally smaller for small particles.

Aerosol particles and droplets with a small Stokes number follow the surrounding fluid and cannot be concentrated efficiently. At high Stokes numbers, the fluid stream fails to re-collimate the particles behind the lens and the stream remains divergent. Liu and co-workers found optimal stokes numbers in the range between $S=0.7$ and 1. They reached good focussing conditions with a 5 lens setup for particles between 20 and $240 \mathrm{~nm}$ diameters.

Aerodynamic lenses have been used successfully for single-shot femtosecond X-ray diffraction of nanoparticles at the soft X-ray free-electron laser FLASH in Hamburg. ${ }^{29}$ In this experiments, ellipsoidal iron oxide nanoparticles with dimensions of $200 \pm 8 \mathrm{~nm}$ and $50 \pm 2 \mathrm{~nm}$ and other nanoparticles have been imaged. The intensity of a single X-ray pulse is sufficient to destroy the particle completely. However, the femtoseconds short pulses of an XFEL source delivers enough scattered photons before radiation damage and melting can destroy the shape of the sample. The aerosol injector that was used in this experiment produced a particle beam of approximately $250 \mu \mathrm{m}$ diameter. ${ }^{34}$ At LCLS, a similar aerodynamic lens injector has been used to image soot particles ${ }^{28}$ and mimi-viruses. ${ }^{13}$

The velocity of the particle stream was between 60 and $150 \mathrm{~m} / \mathrm{s}$. The high speed of the particles is of great importance for the sample refreshment in XFELs. At the European XFEL the X-ray focus will be on the order of $1 \mu \mathrm{m}$ down to $100 \mathrm{~nm}$. At the speed of $60 \mathrm{~m} / \mathrm{s}$ a particle remains less than $20 \mathrm{~ns}$ in the focus area. Even with the highest repetition rate of $4.5 \mathrm{MHz}$ at this XFEL source, the sample is refreshed for the next X-ray pulse.

Equipped with an additional skimmer for differential pumping, these aerodynamic lens injectors are capable to inject particles into a vacuum chamber with an base line pressure between $10^{-5}$ to $10^{-7}$ mbar depending on the samples. If the samples are suspended in solution, the droplets become desolvated on the way through the lens setup. However, adiabatic cooling rapidly cools down the droplets and the samples may end up with a shell of solvent complicating the structure resolution.

\subsection{Native mass spectrometry}

Another option to deliver single particles at XFEL sources is to ionize them as in native mass spectrometry (MS). To transfer intact non-covalent complexes of proteins or other biomolecules into the gas phase of a mass spectrometer, a soft ionisation technique operating with analytes in solution is optimally suited. Therefore, electrospray ionization (ESI, Fig. 2 ) is usually applied in native MS. ${ }^{35-37}$ ESI produces multiply charged ions of single protein complexes (Fig. 2), which reduces the required mass-to-charge ratio $(\mathrm{m} / z)$ to be covered by mass analyzers. From adjacent peaks of an ion series, the mass can be derived. Ions are gradually transferred from atmospheric pressure to vacuum in the source region. Occasional collisions with residual gas especially decrease the transversal velocity of ions and increase the desolvation. Thus, essentially solvent-free protein complexes reach the detector when volatile buffers, such as aqueous ammonium acetate, are used. Resulting signals are often sharp enough to determine accurate binding affinities. ${ }^{38,39}$ Furthermore, ions become trapped in the solution state upon desolvation providing a snapshot of distributions and enabling time-resolved studies. ${ }^{40,41}$

With its capabilities, ESI is superior to aerodynamic lenses. Aerodynamic lenses produce a high background around the sample when the sample size is much smaller than the droplet diameter. The background originates 


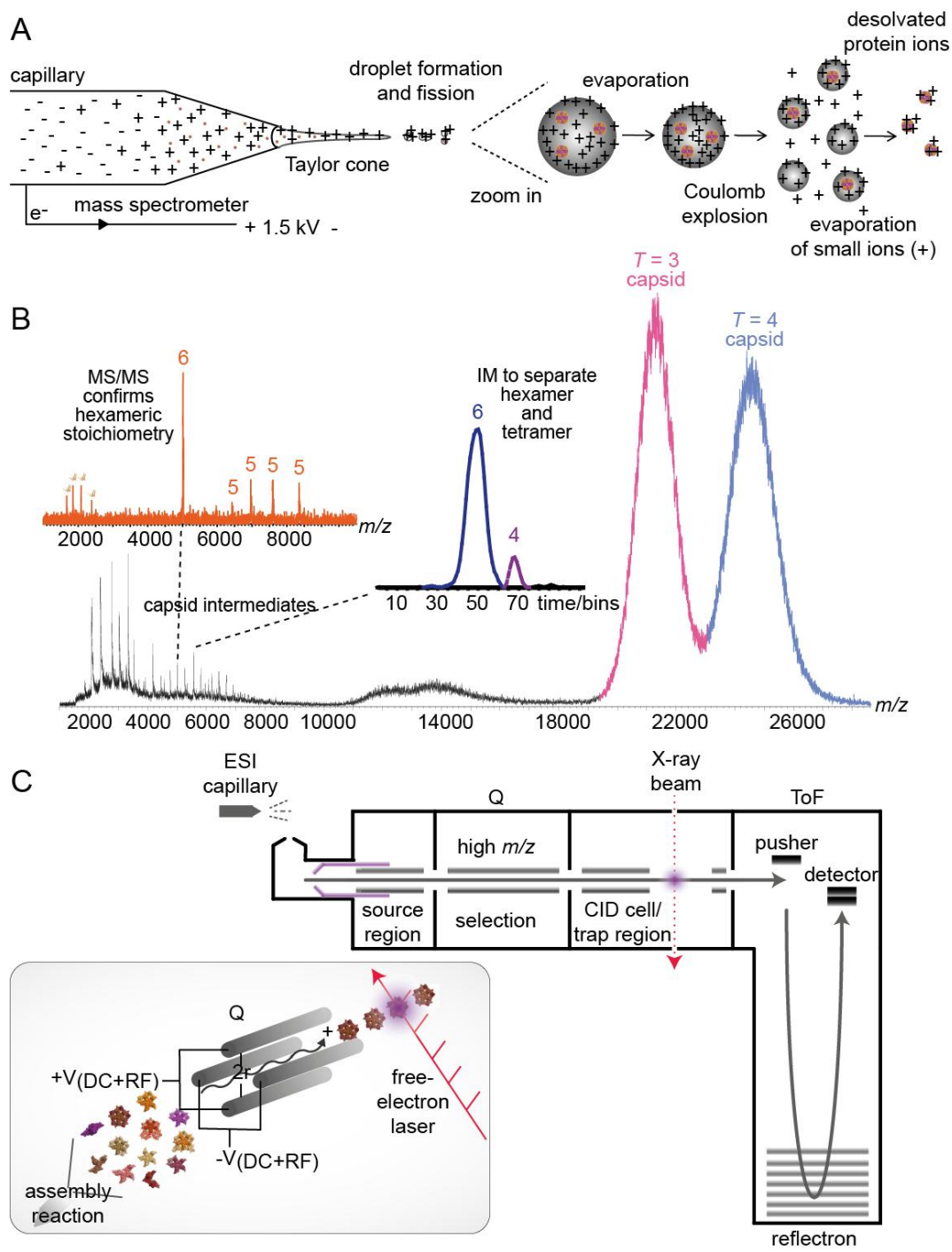

Figure 2. Sample delivery by native mass spectrometry (MS). A) Ionized samples are commonly produced by electrospray ionization (ESI). This method creates small droplets by Coulomb explosion from a shrinking droplet. B)Mass spectrum of hepatitis B virus capsids ${ }^{40,42}$ showing intermediates of the assembly process below $m / z$ 8,000. The left inset shows the selection of a hexameric precursor in the quadrupole (Q) and its identification by subsequent CID. Furthermore, the samples can be shape selected as demonstrated for the $18^{+}$hexamer, which overlaps with the tetramer. C) Scheme of the planned MS setup optimized for XFEL imaging. The interaction with the X-ray from the XFEL is located behind or in an electromagnetic ion-trap. A quadrupole mass selector can assure the selectivity of the injector for a well defined sample. The inset exemplifies this process on a capsid assembly reaction. 
from two sources. First, due to high sample concentration and relatively large droplet sizes, more than one particle or additional material, such as debris from damaged particles, can end up in the same droplet and contribute to the diffraction pattern. Second, residual solvent increases the background scattering. In aerodynamic lenses, the solvent is removed passively by evaporation causing adiabatic cooling. Therefore, evaporation of solvent and buffer components stops below a threshold temperature. Even though, droplets produced in ESI are initially larger than for optimised non-ionising aerosol sources, droplet fission and Coulomb explosion of droplets, triggered by increasing charge during solvent evaporation, lead to production of single particles. Additionally, low sample concentrations ensure separation of particles. Gas collisions of the ions partially compensate adiabatic cooling and ensure complete desolvation.

Of course, a lower sample concentration would also decrease background from additional material in aerodynamic lenses but this would cause a too low XFEL interaction rate for structure determination. This problem can be circumvented in native MS sample delivery as ions even of large protein complexes can be trapped to increase the particle density. ${ }^{43,44}$ The exact location of the interaction region and the trap design are currently investigated to guarantee: sufficient interaction rates, sample refreshment between laser pulses of the European XFEL, removal of plasma components formed after interaction, and recording of diffraction patterns to high resolution. Trapping of ions also enables pulsed particle release, which is difficult to realize in other aerosol sources. Currently, conservative estimates for ion flux created in ESI (over 200,000 protein complexions/s) and density of trapped ions $\left(1000 / \mathrm{mm}^{3}\right)$ suggest that the application of a native mass spectrometer for sample delivery will only be feasible at high repetition rate sources like the European XFEL Facility. At low pulse rates, data collection may not be achieved in a reasonable time frame. In summary, MS can significantly reduce sample consumption and background scattering.

To preserve the structure of a protein complex, recording diffraction patterns directly from solution would be ideal. This can only be achieved with liquid jets, however the surrounding liquid produces a high background with hard X-rays compared to the diffraction intensity of single particles. Therefore, liquid jets are not suited for high resolution structure determination of individual protein complexes. In some cases, the transfer to the gas phase could change the structure of a molecule. This is especially true for flexible regions, however it can be expected that the core of the structure stays intact and biological insights can still be obtained. Furthermore, flexible regions usually are also unresolved in crystals and techniques to protect flexible regions are available in MS. ${ }^{45,46}$

The charge on the ions in MS could further affect the structure. However, the amount of charges per mass unit is low (1 per 2,000 to 20,000 mass units for protein sizes of $20 \mathrm{kDa}$ to $3 \mathrm{MDa}$, respectively). MS results and simulations indicate that solution structures are indeed retained in the gas phase. ${ }^{47,48}$ Ion mobility (IM) allows the shape of ions to be studied, especially monitoring of conformational changes upon ligand binding, ${ }^{49,50}$ albeit with low structural resolution (several $\mathrm{nm}$ ). Results from IM-MS further support the view that the structure is retained. ${ }^{42,44,51-53}$ Even if minor changes occur, significant biological conclusions can probably still be drawn, especially when the protein backbone is unaffected. Resolutions below $1 \mathrm{~nm}$, better $5 \AA$, should be obtained.

After the source, quadrupole mass analyzers (Q, Fig. 2) are often placed to enable selection of ions based on $m / z$ (Fig. 2 insert). These can then be subjected to tandem MS experiments (MS/MS). Single unfolded subunits are usually ejected from the periphery of protein complexes after collision induced dissociation (CID) with rare gases in MS/MS. ${ }^{54} \mathrm{MS} / \mathrm{MS}$ can thereby be utilised to confirm the stoichiometry of a complex and derive topological information. Further topology data can be obtained by adding small amounts of organic modifiers to generate sub-complexes. ${ }^{55}$ Ions from MS and MS/MS mode are finally detected in an orthogonal time-of-flight (ToF) analyzer. We envision placing the trap between the two mass analyzers. This would enable selection of individual species in the Q providing a major advantage compared to aerodynamic lenses, which do not support particle separation by size. Should some debris or other material stick to the particles even in ESI, selection would remove those and only intact protein complexes are imaged. Moreover, purification of protein complexes is often imperfect. The Q can be used to eliminate impurities.

Selectivity in the $\mathrm{Q}$ is of special interest when studying reactions of or between proteins. Reactions represent complex mixtures of start, intermediate and product components. Aerodynamic lenses require that the diffraction patterns are sorted afterwards, which is computationally challenging. Therefore, Q implementation allows imaging of different species separately, significantly reducing the computational costs. The virtual concentration 
of very low abundant or transient species can be increased, which would be missed otherwise. For example in virus capsid assembly, $90 \%$ of the detected signal corresponds to the full capsid and the free building blocks (figure 2B). The remaining $10 \%$ are split amongst the many more interesting intermediates and would probably be missed in the diffraction data without selection. It has been shown that MS with its high sensitivity can detect capsid intermediates. ${ }^{42,56}$ There is also the possibility to insert a mobility analyzer cell prior to the trap and select by shape in addition to $\mathrm{m} / z$.

Particles, which have not interacted with the XFEL, can be detected in the ToF. Thereby, sample influx and integrity are constantly monitored. Maintaining sample supply is a common bottleneck for aerodynamic lenses. Often, the only readout is the production of diffraction patterns. Due to the need for small droplets, tiny jets are used, which are hardly visible in a microscope and often unstable. The other main advantages of ToFs are simultaneous detection of all ion species and a virtually unlimited mass range. Up to now, charge states of protein complexes as heavy as $18 \mathrm{MDa}$ have been resolved and analyzed. ${ }^{57}$

Native MS has so far focussed on soluble proteins from overexpression systems. However, endogenous protein complexes can also be purified, for example by tandem affinity approaches using a tagged component, and subjected to native MS. ${ }^{58,59}$ Due to the high sensitivity of the instruments, the low achieved sample yields are sufficient. Moreover, membrane proteins protected by detergent micelles can be analyzed in native MS. ${ }^{60}$ Stoichiometry and shape are usually retained as for soluble proteins. Furthermore, structural lipids, which usually interact tightly, can be identified in native MS as the detergent cannot replace them. To facilitate mass determination of membrane proteins, the ions are activated in CID to remove the detergent layer from transmembrane regions and resolve charge states.

\section{CONCLUSIONS AND OUTLOOK}

In this proceeding we report the current status of liquid and aerosol sample delivery methods for high repetition rate XFELs and give an outlook to develop a native mass spectrometry into a sample delivery method. Both, liquid jets and aerosol injectors have and will have their place in sample preparation methods for biology.

Liquid jets are successfully used in serial femtosecond crystallography experiments where the Bragg peaks of nano crystals are sufficiently intense to surmount the background scattering from the surrounding solvent. The smallest beam diameters and thereby the best sample efficiency can be reached by gas dynamic virtual nozzles (GDVN). ${ }^{18-20}$

Today, nozzle production is time consuming. Future development is necessary to enable mass production of these devices. Sample efficiency can be further improved for European XFEL by pulsing the sample stream synchronized with the X-ray pulse trains. It is envisioned to develop microfluidic devices for both, mass production of nozzles and synchronized delivery of samples.

Aerodynamic lens stacks are used to bring isolated samples from an aerosol into the X-rays of an XFEL. For single particle imaging, where signals are weak with respect to background scattering from solvents, this is the preferred technology. For hard X-ray imaging, there is demand to focus streams of less than $100 \mathrm{~nm}$ particles. For such small samples, further optimization of the technique is required.

A drawback of aerodynamic lens stacks is their poor sample selectivity. In order to image small particles selectively, we introduce the idea to develop an ion sample delivery system on the basis of native mass spectrometry. This technique is especially useful for structure analysis of protein complexes in their native state. The combination of quadrupole mass spectrometry with an ion trap for storage and pulsed delivery of the selected targets will considerably reduce the background from undesired species and improve the efficiency of sample delivery. For many biologically interesting samples, this is of paramount importance for imaging them with the short and intense X-ray pulses of an XFEL source.

\section{ACKNOWLEDGMENTS}

Charlotte Uetrecht was funded by an EMBO ATLF 1531-2010. 


\section{REFERENCES}

[1] Harmand, M., Coffee, R., Bionta, M. R., Chollet, M., French, D., Zhu, D., Fritz, D. M., Lemke, H. T., Medvedev, N., Ziaja, B., Toleikis, S., and Cammarata, M., "Achieving few-femtosecond time-sorting at hard x-ray free-electron lasers," Nature Photonics 7, 215 (2013).

[2] Altarelli, M., Brinkmann, R., Chergui, M., Decking, W., Dobson, B., Dsterer, S., Grbel, G., Graeff, W., Graafsma, H., Hajdu, J., Marangos, J., Pflger, J., Redlin, H., Riley, D., Robinson, I., Rossbach, J., Schwarz, A., Tiedtke, K., Tschentscher, T., Vartaniants, I., Wabnitz, H., Weise, H., Wichmann, R., Witte, K., Wolf, A., Wulff, M., and Yurkov, M., "The european x-ray free-electron laser, technical design report," Tech. Rep. 2006-097, DESY (2007).

[3] Henrich, B., Becker, J., Dinapoli, R., Goettlicher, P., Graafsma, H., Hirsemann, H., Klanner, R., Krueger, H., Mazzocco, R., Mozzanica, A., Perrey, H., Potdevin, G., Schmitt, B., Shi, X., Srivastava, A., Trunk, U., and Youngman, C., "The adaptive gain integrating pixel detector agipd a detector for the european xfel," Nucl. Instr. and Meth. A 633, S11 (2011).

[4] Blue, A., French, M., Seller, P., and OShea, V., "Edgeless sensor development for the lpd hybrid pixel detector at xfel," Nucl. Instr. and Meth. A 1, 55 (2009).

[5] Coughlan, J., Cook, S., Day, C., Halsall, R., and Taghavi, S., "The data acquisition card for the large pixel detector at the european-xfel," Journal of Instrumentation 6, C12057 (2011).

[6] Wabnitz, H., Bittner, L., De Castro, A., Döhrmann, R., Gürtler, P., Laarmann, T., Laasch, W., Schulz, J., Swiderski, A., von Haeften, K., et al., "Multiple ionization of atom clusters by intense soft x-rays from a free-electron laser," Nature 420, 482 (2002).

[7] Young, L., Kanter, E. P., Krässig, B., Li, Y., March, A. M., Pratt, S. T., Santra, R., Southworth, S. H., Rohringer, N., DiMauro, L. F., et al., "Femtosecond electronic response of atoms to ultra-intense x-rays," Nature 466, 56 (2010).

[8] Gorkhover, T., Adolph, M., Rupp, D., Schorb, S., Epp, S., Erk, B., Foucar, L., Hartmann, R., Kimmel, N., Kuühnel, K.-U., et al., "Nanoplasma dynamics of single large xenon clusters irradiated with superintense x-ray pulses from the linac coherent light source free-electron laser," Physical Review Letters 108, 245005 (2012).

[9] Neutze, R., Wouts, R., van der Spoel, D., Weckert, E., and Hajdu, J., "Potential for biomolecular imaging with femtosecond x-ray pulses," Nature 406, 752 (2000).

[10] Chapman, H. N., Barty, A., Bogan, M. J., Boutet, S., Frank, M., Hau-Riege, S. P., Marchesini, S., Woods, B. W., Bajt, S., and others, W. H. B., "Femtosecond diffractive imaging with a soft-x-ray free-electron laser," Nature Physics 2, 839 (2006).

[11] Chapman, H., Fromme, P., Barty, A., White, T., Kirian, R., Aquila, A., Hunter, M., Schulz, J., DePonte, D., Weierstall, U., et al., "Femtosecond x-ray protein nanocrystallography," Nature 470, 73-77 (2011).

[12] Redecke, L., Nass, K., DePonte, D. P., White, T. A., Rehders, D., Barty, A., Stellato, F., Liang, M., Barends, T. R., Boutet, S., et al., "Natively inhibited trypanosoma brucei cathepsin b structure determined by using an x-ray laser," Science 339, 227 (2013).

[13] Seibert, M. M., Ekeberg, T., Maia, F. R. N. C., Svenda, M., Andreasson, J., Jönsson, O., Odic, D., Iwan, B., Rocker, A., Westphal, D., et al., "Single mimivirus particles intercepted and imaged with an x-ray laser," Nature 470, 78 (2011).

[14] Siefermann, K. R., Liu, Y., Lugovoy, E., Link, O., Faubel, M., Buck, U., Winter, B., and Abel, B., "Binding energies, lifetimes and implications of bulk and interface solvated electrons in water," Nature Chemistry $\mathbf{2}$, $274(2010)$.

[15] Winter, B. and Faubel, M., "Photoemission from liquid aqueous solutions," Chem. Rev. 106, 1176 (2006).

[16] Kleinekofort, W., Avdiev, J., and Brutschy, B., "A new method of laser desorption mass spectrometry for the study of biological macromolecules," International journal of mass spectrometry and ion processes $\mathbf{1 5 2}$, 135 (1996).

[17] Faubel, M., Siefermann, K. R., Liu, Y., and Abel, B., "Ultrafast soft x-ray photoelectron spectroscopy at liquid water microjets," Accounts of chemical research 45, 120 (2012).

[18] Montanero, J. M., Rebollo-Munoz, N., Herrada, M. A., and Ganan-Calvo, A. M., "Global stability of the focusing effect of fluid jet flows," Physical Review E 83, 036309 (2011). 
[19] DePonte, D. P., Weierstall, U., Schmidt, K., Warner, J., Starodub, D., Spence, J. C. H., and Doak, R. B., "Gas dynamic virtual nozzle for generation of microscopic droplet streams," J. Phys. D 41, 195505 (2008).

[20] Weierstall, U., Spence, J. C. H., and Doak, R. B., "Injector for scattering measurements on fully solvated biospecies," Review of scientific instruments 83, 035108 (2012).

[21] DePonte, D. P., Mckeown, J. T., Weierstall, U., Doak, R. B., and Spence, J. C. H., "Towards etem serial crystallography: Electron diffraction from liquid jets," Ultramicroscopy 111, 824 (2011).

[22] Charvat, A., Lugovoj, E., Faubel, M., and Abel, B., "New design for a time-of-flight mass spectrometer with a liquid beam laser desorption ion source for the analysis of biomolecules," Review of scientific instruments 75, 1209 (2004).

[23] Lomb, L., Steinbrenner, J., Bari, S., Beisel, D., Berndt, D., Kieser, C., Lukat, M., Neef, N., and Shoeman, R., "An anti-settling sample delivery instrument for serial femtosecond crystallography," Journal of applied crystallography 45, 674 (2012).

[24] Dorman, R. G., "The atomization of liquid in a flat spray," British journal of applied physics 3, 189 (1952).

[25] Villermaux, E. and Clanet, C., "Life of a flapping liquid sheet," Journal of fluid mechanics 462, 341 (2002).

[26] Li, R. and N., A., "Characteristics of liquid sheets formed by two impinging jets," Phys. Fluids 18, 087104 (2006).

[27] Clanet, C., "Waterbells and liquid sheets," Annual review of fluid mechanics 39, 469 (2007).

[28] Martin, A. V., Loh, N. D., Hampton, C. Y., Sierra, R. G., Wang, F., Aquila, A., Bajt, S., Barthelmess, M., Bostedt, C., Bozek, J. D., et al., "Femtosecond dark-field imaging with an x-ray free electron laser," Optics Express 20, 13501 (2012).

[29] Bogan, M. J., Boutet, S., Barty, A., Benner, W. H., Frank, M., Lomb, L., Shoeman, R., Starodub, D., Seibert, M. M., Hau-Riege, S. P., Woods, B., Decorwin-Martin, P., Bajt, S., Schulz, J., Rohner, U., Iwan, B., Timneanu, N., Marchesini, S., Schlichting, I., Hajdu, J., and Chapman, H. N., "Single-shot femtosecond x-ray diffraction from randomly oriented ellipsoidal nanoparticles," Phys Rev Special Topics - Accelerators and Beams 13, 094701 (2010).

[30] Fienup, J. R., "Reconstruction of a complex-valued object from the modulus of its fourier transform using a support constraint," J. Opt. Soc. Am. A 4, 118 (1987).

[31] Murphy, W. K. and Sears, G. W., "Production of particulate beams," J. Appl. Phys. 35, 1986 (1964).

[32] Liu, P., Ziemann, P. L., Kittelson, D. B., and McMurry, P. H., "Generating particle beams of controlled dimensions and divergence: I. theory of particle motion in aerodynamic lenses and nozzle expansions," Aerosol Science and Technology 22(3), 293 (1995).

[33] Liu, P., Ziemann, P. L., Kittelson, D. B., and McMurry, P. H., "Generating particle beams of controlled dimensions and divergence: Ii. experimental evaluation of particle motion in aerodynamic lenses and nozzle expansions," Aerosol Science and Technology 22(3), 314 (1995).

[34] Benner, W. H., Bogan, M. J., Rohner, U., Boutet, S., Woods, B., and Frank, M., "Non-destructive characterization and alignment of aerodynamically focussed particle beams using single particle charge detection," Journal of Aerosol Science 39, 917 (2008).

[35] Benesch, J. L., Ruotolo, B. T., Simmons, D. A., and Robinson, C. V., "Protein complexes in the gas phase: technology for structural genomics and proteomics," Chem Rev 107, 3544-67 (2007).

[36] Lorenzen, K. and van Duijn, E., "Native mass spectrometry as a tool in structural biology," Curr Protoc Protein Sci Chapter 17, Unit17.12 (2010).

[37] Heck, A. J., "Native mass spectrometry: a bridge between interactomics and structural biology," Nat Methods 5, 927 (2008).

[38] Rose, R. J., Labrijn, A. F., van den Bremer, E. T., Loverix, S., Lasters, I., van Berkel, P. H., van de Winkel, J. G., Schuurman, J., Parren, P. W., and Heck, A. J., "Quantitative analysis of the interaction strength and dynamics of human igg4 half molecules by native mass spectrometry.," Structure 19, 1274-82 (2011).

[39] El-Hawiet, A., Kitova, E. N., Arutyunov, D., Simpson, D. J., Szymanski, C. M., and Klassen, J. S., "Quantifying ligand binding to large protein complexes using electrospray ionization mass spectrometry," Anal Chem 84, 3867-70 (2012). 
[40] Uetrecht, C., Versluis, C., Watts, N. R., Roos, W. H., Wuite, G. J., Wingfield, P. T., Steven, A. C., and Heck, A. J., "High-resolution mass spectrometry of viral assemblies: molecular composition and stability of dimorphic hepatitis b virus capsids," Proc Natl Acad Sci USA 105, 9216-20 (2008).

[41] Brettschneider, C., Rose, R. J., Hertel, S., Axmann, I. M., Heck, A. J., and Kollmann, M., "A sequestration feedback determines dynamics and temperature entrainment of the kaiabc circadian clock," Mol Syst Biol 6, 389 (2010).

[42] Uetrecht, C., Barbu, I. M., Shoemaker, G. K., van Duijn, E., and Heck, A. J., "Interrogating viral capsid assembly with ion mobility mass spectrometry," Nat Chem 3, 126 (2011).

[43] Pringle, S. D., Giles, K., Wildgoose, J. L., Williams, J. P., Slade, S. E., Thalassinos, K., Bateman, R. H., Bowers, M. T., and Scrivens, J. H., "An investigation of the mobility separation of some peptide and protein ions using a new hybrid quadrupole/travelling wave ims/oa-tof instrument," Int. J. Mass Spectrom. 261, 1 (2007).

[44] Uetrecht, C., Versluis, C., Watts, N. R., Wingfield, P. T., Steven, A. C., and Heck, A. J., "Stability and shape of hepatitis b virus capsids in vacuo," Angew Chem Int Ed Engl 47(33), 6247-51 (2008).

[45] Warnke, S., von Helden, G., and Pagel, K., "Protein structure in the gas phase: The influence of side-chain microsolvation," J. Am. Chem. Soc. 135, 117 (2013).

[46] Pagel, K., Natan, E., Hall, Z., R., F. A., and V., R. C., "Intrinsically disordered p53 and its complexes populate compact conformations in the gas phase," Angewandte Chemie 125, 379 (2013).

[47] Uetrecht, C. and Heck, A. J., "Modern biomolecular mass spectrometry and its role in studying virus structure, dynamics and assembly," Angew. Chem. Int. Ed. Engl. 50, 8248 (2011).

[48] Marklund, E. G., Larsson, D. S., van der Spoel, D., Patriksson, A., and Caleman, C., "Structural stability of electrosprayed proteins: temperature and hydration effects," Phys Chem Chem Phys 11, 8069 (2009).

[49] Alverdi, V., Mazon, H., Versluis, C., Hemrika, W., Esposito, G., van den Heuvel, R., Scholten, A., and Heck, A. J., "cgmp-binding prepares pkg for substrate binding by disclosing the c-terminal domain," $J$ Mol Biol 375, 1380 (2008).

[50] Uetrecht, C., Rose, R. J., Van Duijn, E., Lorenzen, K., and Heck, A. J. R., "Ion mobility mass spectrometry of proteins and protein assemblies," Chemical Society Reviews 39(5), 1633-1655 (2010).

[51] Ruotolo, B. T., Giles, K., Campuzano, I., Sandercock, A. M., Bateman, R. H., and Robinson, C. V., "Evidence for macromolecular protein rings in the absence of bulk water," Science 310, 1658 (2005).

[52] van Duijn, E., Barendregt, A., Synowsky, S., Versluis, C., and Heck, A. J., "Chaperonin complexes monitored by ion mobility mass spectrometry," J. Am. Chem. Soc. 131, 1452 (2009).

[53] Knapman, T. W., Morton, V. L., Stonehouse, N. J., Stockley, P. G., and Ashcroft, A. E., "Determining the topology of virus assembly intermediates using ion mobility spectrometry-mass spectrometry," Rapid Commun Mass Spectrom 24, 3033 (2010).

[54] Lorenzen, K., Vannini, A., Cramer, P., and Heck, A. J., "Structural biology of rna polymerase iii: Mass spectrometry elucidates subcomplex architecture," Structure 15, 1237 (2007).

[55] Jore, M. M., Lundgren, M., van Duijn, E., Bultema, J. B., Westra, E. R., Waghmare, S. P., Wiedenheft, B., Pul, U., Wurm, R., Wagner, R., Beijer, M. R., Barendregt, A., Zhou, K., Snijders, A. P., Dickman, M. J., Doudna, J. A., Boekema, E. J., Heck, A. J., van der Oost, J., and Brouns, S. J., "Structural basis for crispr rna-guided dna recognition by cascade," Nat Struct Mol Biol 18, 529 (2011).

[56] Shoemaker, G. K., van Duijn, E., Crawford, S. E., Uetrecht, C., Salumbides-Baclayon, M., Roos, W. H., Wuite, G. J., Estes, M. K., Prasad, B. V., and Heck, A. J., "Norwalk virus assembly and stability monitored by mass spectrometry," Mol Cell Proteomics 9, 1742 (2010).

[57] Snijder, J., Rose, R. J., Veesler, D., Johnson, J. E., and Heck, A. J., "Studying 18 mda virus assemblies with native mass spectrometry," Angew Chem Int Ed Engl 0, 0 (2013).

[58] Synowsky, S. A., van Wijk, M., Raijmakers, R., and Heck, A. J., "Comparative multiplexed mass spectrometric analyses of endogenously expressed yeast nuclear and cytoplasmic exosomes," J Mol Biol 385, 1300-13 (2009).

[59] Synowsky, S. A. and Heck, A. J., "The yeast ski complex is a hetero-tetramer," Protein Sci 17, 119 (2008).

[60] Barrera, N. P., Zhou, M., and Robinson, C. V., "The role of lipids in defining membrane protein interactions: insights from mass spectrometry," Trends Cell Biol 23, 1 (2013). 